\title{
The SKA and Galaxy Cluster Science with the Sunyaev-Zel'dovich Effect
}

\author{
Keith Grainge ${ }^{1}$, Stefano Borgani ${ }^{2,3}$, Sergio Colafrancesco ${ }^{4}$, Chiara Ferrari ${ }^{5}$, Anna \\ Scaife $^{6}$, Paolo Marchegiani ${ }^{4}$, S. Emritte ${ }^{4}$. J. Weller ${ }^{7,8,9}$ \\ ${ }^{1}$ Jodrell Bank Centre for Astrophysics, School of Physics and Astronomy, The University of \\ Manchester, Manchester, UK; \\ ${ }^{2}$ Astronomy Unit, Department of Physics, University of Trieste, via G.B. Tiepolo 11, I-34131 \\ Trieste, Italy; \\ ${ }^{3}$ INFN-National Institute for Nuclear Physics, Via Valerio 2, I-34127 Trieste, Italy; \\ ${ }^{4}$ School of Physics, University of the Witwatersrand, 1 Jan Smut Ave, WITS-2050 Johannesburg, \\ South Africa; \\ ${ }^{5}$ Laboratoire Lagrange, UMR 7293, Université de Nice Sophia-Antipolis, CNRS, Observatoire \\ de la Côte d'Azur, 06300 Nice, France; \\ ${ }^{6}$ School of Physics \& Astronomy, University of Southampton, Southampton, SO17 1BJ, UK; \\ ${ }^{7}$ University Observatory Munich, Department of Physics, Ludwig-Maximilians University, \\ 81679 Munich, Germany; \\ ${ }^{8}$ Excellence Cluster Universe, Boltzmannstr. 2, 85748 Garching bei Munchen, Germany; \\ ${ }^{9}$ Max-Planck-Institute for Extraterrestrial Physics, Giessenbachstrasse, 85748 Garching, \\ Germany \\ E-mail: keith.grainge@manchester.ac.uk
}

Studying galaxy clusters through their Sunyaev-Zel'dovich (SZ) imprint on the Cosmic Microwave Background has many important advantages. The total SZ signal is an accurate and precise tracer of the total pressure in the intra-cluster medium and of cluster mass, the key observable for using clusters as cosmological probes. Band 5 observations with SKA-MID towards cluster surveys from the next generation of X-ray telescopes such as e-ROSITA and from Euclid will provide the robust mass estimates required to exploit these samples. This will be especially important for high redshift systems, arising from the SZ's unique independence to redshift. In addition, galaxy clusters are very interesting astrophysical systems in their own right, and the SKA's excellent surface brightness sensitivity down to small angular scales will allow us to explore the detailed gas physics of the intra-cluster medium.

Advancing Astrophysics with the Square Kilometre Array

June 8-13, 2014

Giardini Naxos, Sicily, Italy 


\section{Introduction}

The Sunyaev-Zel'dovich (SZ) effect (Sunyaev \& Zel'dovich 1970,1972) is a secondary anisotropy introduced onto the Cosmic Microwave Background (CMB) through inverse Compton scattering of CMB photons from the electrons (thermal and non-thermal) contained the intra-cluster medium (ICM) of galaxy clusters (see e.g. Birkinshaw (1999); Carlstrom et al. (2002) for an overview of the SZ effect). On average this scattering leads to an increase in the energy of these photons, while conserving photon number, which results in a change in intensity $\Delta I_{v}$ from that of the CMB

$$
\Delta I_{v}=\frac{2\left(k_{B} T_{0}\right)^{3}}{(h c)^{2}} \frac{\sigma_{T}}{m_{e} c^{2}} \int P_{e} g(x) d l
$$

where $T_{0}$ is the temperature of the CMB today, $h$ is the Planck constant, $k_{B}$ is the Boltzmann constant and $c$ is the speed of light. $P_{e}$ is the electron pressure in the cluster ICM (i.e. $P_{e}=k_{B} T_{e} \cdot n_{e}$ for a thermal plasma with temperature $T_{e}$ and number density $n_{e}$ of the scattering electrons); and $g(x)$ is the spectral shape of the SZ effect (see Figure 1), in the non-relativistic limit given by

$$
g_{n r}(x)=\frac{x^{4} e^{x}}{\left(e^{x}-1\right)^{2}}\left[x \operatorname{coth} \frac{x}{2}-4\right]
$$

in terms of the non-dimensional frequency $x$ given by

$$
x=\frac{h v}{k_{B} T_{0}}
$$

SKA1-MID will have the capability to spectrally separate thermal and non-thermal components of the SZ effect.
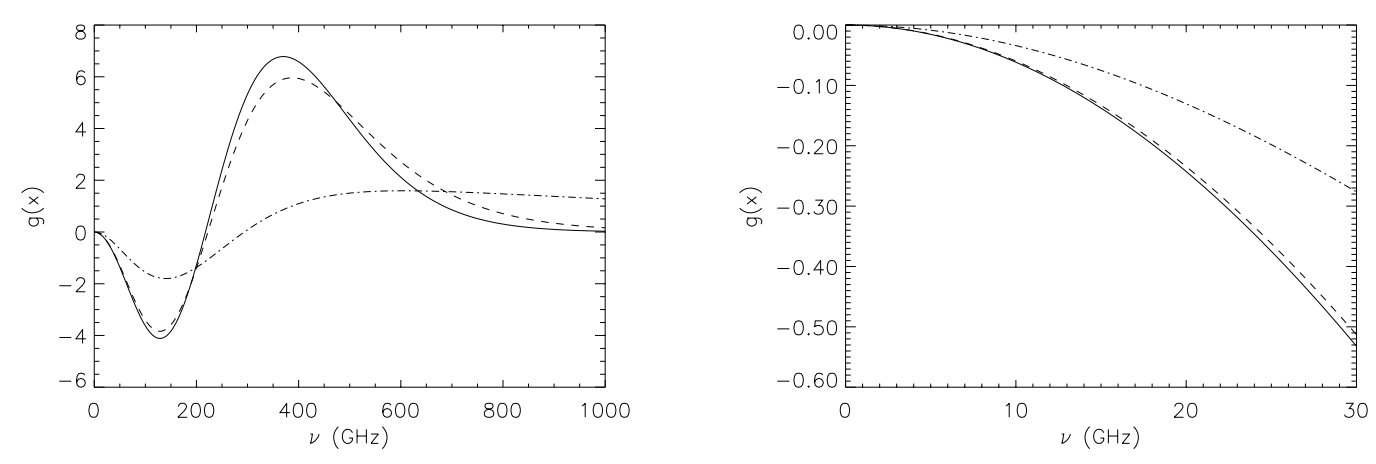

Figure 1: Left. The spectral function $g(x)$ calculated for a cluster with temperature $k_{B} T_{e}=10 \mathrm{keV}$ (dashed) compare to the non-relativistic case (solid). We also show the spectral function for a relativistic, non-thermal plasma with spectrum $N_{e, r e l} \propto E^{-3.5}$ and $E_{e, \min }=722.7 \mathrm{keV}$ (dot-dashed). Right. A zoom of the same plot in the frequency range up to $30 \mathrm{GHz}$, which is the most relevant for the SKA. (Colafrancesco et al. 2003)

Equation 1.1 shows the following key aspects which make the SZ effect a powerful probe of galaxy clusters:

- The surface brightness of the SZ effect is independent of redshift, $z$. The cosmological dimming of radiation by $(1+z)^{4}$ due to the expansion of the Universe is exactly cancelled 
by the increased CMB energy density at the time of the scattering. It is therefore possible to see the SZ effect from clusters all the way back to the epoch of their formation, provided that they retain their ICM.

- The intensity of the SZ effect is proportional to the line-of-sight integral of the total pressure of the ICM. Therefore the integrated SZ flux over solid angle is proportional to the total thermal energy in the cluster, which is expected to be closely related to the overall cluster mass. This point will be returned to in Section 2.

At frequencies below the null of the SZ effect (which is found at $217 \mathrm{GHz}$ in the nonrelativistic limit) the SZ effect shows an intensity decrement towards the cluster, a very useful characteristic signature for discriminating the SZ signal from other emission mechanisms. At centimetre radio frequencies we are in the Rayleigh-Jeans regime and $I_{v}$ is well approximated as having a thermal $v^{2}$ frequency dependence. Given that contaminating radio halo emission typically has a falling $v^{-\alpha_{r}}$ spectrum with $\alpha_{r}>1$ (Feretti et al. 2012), it is often advantageous to perform SZ work at high frequency (e.g. SPT (Carlstrom et al. 2011); ACT (Swetz et al. 2011); Planck (Planck Collaboration XI 2011)). However, both the first reliable SZ detection (Birkinshaw et al. 1984) and the first SZ image (Jones et al. 1993) were made at $15 \mathrm{GHz}$; and this same frequency was used by one of the next generation of telescopes specifically designed for SZ work, AMI (Zwart et al. 2008), which has made follow-up detections of 99 Planck clusters (Perrott et al. 2014). Based on these observations, in this Chapter we explore the possibility of observing the SZ-effect from galaxy clusters using SKA operating in Band 5. We focus upon improving knowledge of cluster scaling relations, which are key to exploiting clusters as tracers for cosmology; and upon probing the detailed astrophysics of the intracluster plasma.

\section{Cluster masses with SKA SZ observations}

Clusters of galaxies are recognised as powerful cosmological probes (Allen et al. 2011; Kravtsov $\&$ Borgani 2012). Measurement of their number counts constrain cosmological parameters through the sensitive dependence of halo mass function to the linear growth rate of cosmic density perturbations. Furthermore, under the assumption that clusters are fair containers of cosmic baryons, measurements of the redshift dependence of their gas mass fraction provide tight constraints on cosmological parameters through the expansion history.

Both such cosmological applications of galaxy clusters necessarily require precise measurements of the total cluster mass and a precise characterisation of the physical properties of the intra-cluster medium. Since direct mass measurements are rather difficult to carry out for a large ensemble of galaxy clusters, a convenient approach for the cosmological exploitation of large cluster surveys should be based on the measurement of suitable mass proxies which are at the same time relatively easy to infer from observations and tightly related to cluster mass.

As already mentioned in the Introduction, the total SZ signal of a galaxy cluster is proportional to the total thermal energy content of the ICM. In fact, for a cluster at redshift $z$, the integrated SZ signal within an aperture angle $\theta$ can be written as

$$
Y(\theta)=D_{A}(z)^{-2} Y(R)=2 \pi D_{A}(z)^{-2} \int_{0}^{R} y(r) r d r
$$


where $D_{A}(z)$ is the angular diameter distance and $y(r)$ is the profile of the Comptonisation parameter. This quantity is proportional to the line-of-sight integral of the electron pressure, which is $n_{e} T_{e}$ for a thermal plasma, according to

$$
y=\int \sigma_{T} n_{e} \frac{k_{B} T_{e}}{m_{e} c^{2}} d l
$$

Therefore, as long as the ICM can be described as a plasma in hydrostatic equilibrium within the cluster potential well, we expect the integrated SZ signal to be tightly related to the total cluster mass. Under the further assumption that gas follows the DM distribution, the prediction of the self-similar model (see, e.g., Arnaud et al. (2010)) gives

$$
Y_{\Delta_{c}} \propto E(z)^{2 / 3} M_{\Delta_{c}}^{5 / 3}
$$

for the scaling relation between mass and integrated $Y$ parameter, both computed within an aperture encompassing an overdensity of $\Delta_{c}$ times the critical density at the cluster redshift, $\rho_{c}(z)$. In the above equation, $E(z)$ describes the redshift dependence of the Hubble parameter.

As a consequence, the total SZ signal provides a rather precise and robust mass proxy. It is precise, since its scaling relation against cluster total mass is characterized by a small intrinsic scatter. It is robust, since this scaling relation has a quite weak dependence on the physical processes which determine the ICM thermodynamical properties. This is illustrated in Figure 2, which shows results on the $Y-M$ scaling at $\Delta_{c}=500$ from a set of hydrodynamical simulations of galaxy clusters (see also Kay et al. (1999); Sembolini et al. (2013); Le Brun et al. (2014)), compared to observational results (from Fabian et al. in prep). The results from simulations clearly show that the scaling relation between integrated SZ signal and mass has a low scatter, $<10 \%$, with slope and normalisation which are almost independent of the physical processes included in the simulations.

Future surveys, both in the X-rays (e.g. from eROSITA ${ }^{1}$ (Pillepich et al. 2012)) or in optical/nearIR band (i.e. LSST $^{2}$ and Euclid ${ }^{3}$ ) will detect $\sim 10^{5}$ clusters and groups over an area of about $10^{4}$ $\mathrm{deg}^{2}$. Mass measurements in the X-rays will be limited to a small fraction (few thousands) of mostly nearby clusters from eROSITA, while weak lensing mass measurements in the Euclid survey will be mostly limited to $z<1$ clusters.

Thanks to its sensitivity, SKA1-MID will allow targeted follow-up observation of clusters detected at high redshift in these future surveys, thereby providing mass measurements, through a calibration of the $Y-M$ relation, for the $z>1$ cluster population.

At the same time, the high angular resolution accessible by SKA1-MID will allow accurate measurement of ICM pressure profiles. The verification of the hydrostatic equilibrium condition to these pressure profiles will allow reconstruction of mass profiles in a robust way. It is worth pointing out that pressure profiles through X-ray observations (Arnaud et al. 2010; Sun et al. 2011) are obtained in an indirect way from the combination of surface gas mass and temperature profiles. Gas clumping is expected to bias the X-ray measurements of both gas mass profiles from surface brightness profiles and temperature profiles because the thermal bremsstrahlung X-ray emissivity

\footnotetext{
${ }^{1}$ http://www.mpe.mpg.de/eROSITA

${ }^{2} \mathrm{http}: / /$ www.lsst.org/lsst/

${ }^{3}$ http://sci.esa.int/euclid/
} 


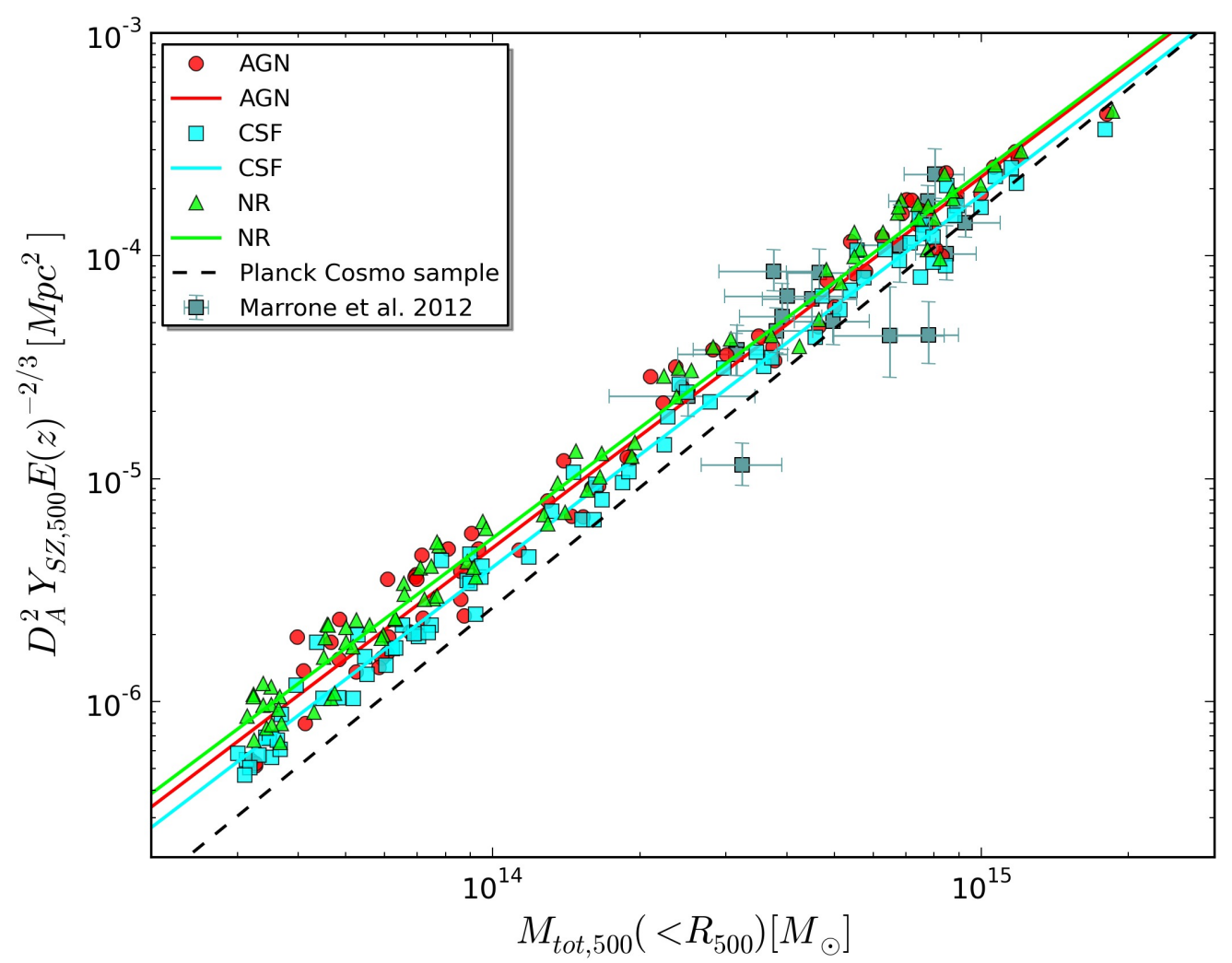

Figure 2: The relation between total mass and integrated SZ signal, both computed within an aperture encompassing at overdensity $\Delta_{c}=500$ times the critical density, for both simulations and observational data (after Fabjan et al. 2014, in preparation). Points without errorbars refer to results from a set of cosmological hydrodynamical simulations of galaxy clusters (Planelles et al. 2014) (green triangles: non-radiative simulations; cyan squares: simulations including radiative cooling, star formation and supernova feedback; red circles: further including AGN feedback). Gray squares with errorbars are observational results from Marrone et al. (2012), while the dashed black line is the best-fit to the observational calibration presented in Planck Collaboration XX (2013).

$\varepsilon_{b r e m} \propto n_{e}^{2} T^{1 / 2}$. On the other hand, the tendency of gas to sit in pressure equilibrium is such that gas clumping should have a minor impact on pressure profiles, that are directly measured by highresolution SZ observations, in which the CMB temperature decrease is $\Delta T_{C M B} \propto n_{e} T_{e}$.

Thanks to the different dependencies of $\mathrm{X}$-ray and SZ signals on gas density and temperature, their combination offers the possibility to measure these two quantities without resorting to challenging X-ray spectroscopy. This possibility is quite interesting in view of the all-sky X-ray survey to be provided by eROSITA. Owing to the relatively shallow flux limit of the eROSITA survey, only surface brightness profiles will be available for most of the clusters that will be detected in this survey at relatively high signal-to-noise ratio. The combination of these X-ray observations with SZ maps from SKA1-MID will allow one to recover electron pressure profiles and hence the temperature profiles deconvolved with information coming from the density profiles.

eROSITA is predicted to have a cluster mass detection limit of $M_{200}=4 \times 10^{14} M_{\odot}$ at $z>1$ 


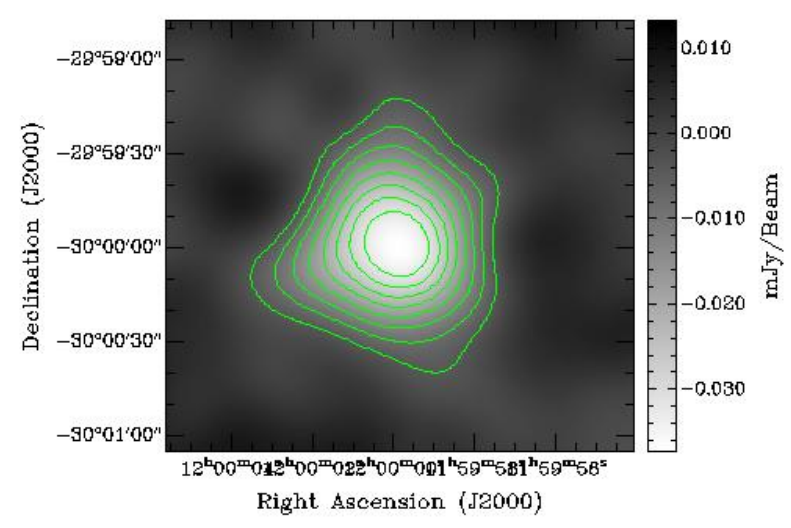

Figure 3: Simulated 1-hour observation with SKA1-MID of a cluster with $M_{200}=4 \times 10^{14} M_{O}$ at $z=1.83$ mapped with a $5 k \lambda$ uv-taper. The cluster SZ effect is detected here at $14 \sigma$ c.l.

and will detect approximately 1000 clusters at $\mathrm{z}>1$ and 10 at $\mathrm{z}>1.83$ (Merloni et al. 2012). Figure 3 demonstrates the capability of SKA1-MID to follow-up these clusters in SZ; it shows a mock observation of a $M_{200}=4 \times 10^{14} M_{\odot}$ cluster observed for one hour by SKA-MID in band 5 (8.8$13.8 \mathrm{GHz}$ ) and is able to detect the SZ effect at $14 \sigma$. Since the SZ effect is an extended feature, we apply a $5 k \lambda$ uv-taper as a crude matched filter. The data from the long SKA1-MID baselines are therefore effectively discarded, but as discussed further in Section 5 these data are used for the removal of contamination from radio point sources. This simulation demonstrates that a 1000-hour SKA1-MID programme can therefore follow up all of the high redshift sample that eROSITA will discover. In addition these additional observations of mass proxies will allow a better estimate of the scatter in the mass-observable relation for clusters detected with Euclid (Rozo et al. 2009).

\section{Detailed investigations of the intra-cluster medium}

Deep X-ray observations of galaxy clusters have revealed different kind of structures in the density and temperature distribution of the ICM, from central X-ray cavities filled with the radio emitting relativistic plasma ejected by active galaxies (e.g. Fabian et al. (2000)), to high surface brightness regions, such as shock and cold fronts related to cluster mergers (e.g. Markevitch (2008) and references therein). Detailed studies of the ICM pressure distribution are necessary to characterise the complex dynamical and feedback processes acting within galaxy clusters.

The SZ effect surface brightness provides a direct measure of the integrated pressure along the line of sight and is well suited to identifying ICM discontinuities (such as cold fronts or shocks) in the absence of resolved X-ray spectroscopy. High-sensitivity and high-resolution SZ observations are therefore an extremely valuable tool to study the evolutionary physics of the ICM.

In the 2030 horizon, SKA2 will be ideally complemented by the Athena X-ray satellite ${ }^{4}$ for the study of high-redshift clusters. Thanks to its large collecting area and spectroscopic capability, Athena will open the possibility of studying the thermal and dynamical status of the ICM at

\footnotetext{
${ }^{4}$ http://www.the-athena-x-ray-observatory.eu
} 
unprecedented precision for a significant number of galaxy clusters. A combination of X-ray observations from Athena with detailed electron pressure maps from SKA2 will shed light on the thermal structure of the ICM and on the dynamics of gas motions associated to the hierarchical build-up of galaxy clusters and by AGN feedback processes taking place in the cluster core regions.

In the last years increasing attention is being paid to the analysis of diffuse intracluster radio emission related to the presence of a non-thermal component in the ICM, with the main aim of studying its link with the complex evolutionary physics of galaxy clusters (e.g. Ferrari et al. (2008); Feretti et al. (2012); Brunetti \& Jones (2014)). A joint analysis of synchrotron emission and SZ signal is emerging as a promising tool to study the interplay between the non-thermal and thermal component of the ICM (see, e.g., Basu (2012); Cassano et al. (2013); Colafrancesco et al. (2014) and references therein). In this context, combined GMRT observations (at 610 and $240 \mathrm{MHz}$ ) with high-resolution MUSTANG results on the galaxy cluster RXJ1347, (Ferrari et al. 2011) have pointed out a strong correlation between an excess in the radio surface brightness of the diffuse radio source at the centre of the cluster (a radio mini-halo already detected by Gitti et al. (2007)) and a high pressure region detected in the SZ map of RX J1347 and confirmed by X-ray observations (see Fig. 4). This result indicates that, in addition to the relativistic electrons ejected by the AGN and (possibly) re-accelerated by MHD turbulence in the central cluster region, the presence of cosmic rays in the excess emission of the radio mini-halo is most likely related to a shock front propagating into the ICM. Figure 5 shows a simulated 1-hour observation of cluster RXJ1347 with the SKA1-MID.

These examples aim at illustrating that, if Band 5 will be covered by the MID component, SKA will be the first telescope able to efficiently detect both synchrotron and SZ radiation, thus becoming an extremely powerful instrument for getting a complete view of the thermal and nonthermal physics of the ICM.

\section{SKA1-MID observations of cluster SZ signals}

Galaxy clusters typically have a size $>1 \mathrm{Mpc}$. The observed SZ signal will therefore be heavily resolved out on long SKA1-MID baselines. However, the compact core of SKA1-MID has a high filling factor and this is critical for measuring low surface brightness features such as the SZ effect.

The simulations presented in this chapter use only the SKA1-MID's cross-correlation visibilities. If, in addition, auto-correlation data are available, these will give the zero-spacing SZ flux and this will aid immensely in inferring an accurate reconstruction of the ICM properties. The expected statistical significance of the auto-correlation data corresponding to the 1-hour observation in Figure 3 is $40 \sigma$, while that in Figure 5 is $300 \sigma$.

\section{Removal of contaminating signals}

It is expected that there will be substantial contamination of the SZ signal from discrete radio sources in the field, but this contamination can be removed by a technique described in e.g. Grainge et al. (1993). The flux and positions of the point sources can be measured with high precision by mapping with the long SKA1-MID baselines, which are much more numerous than the short 

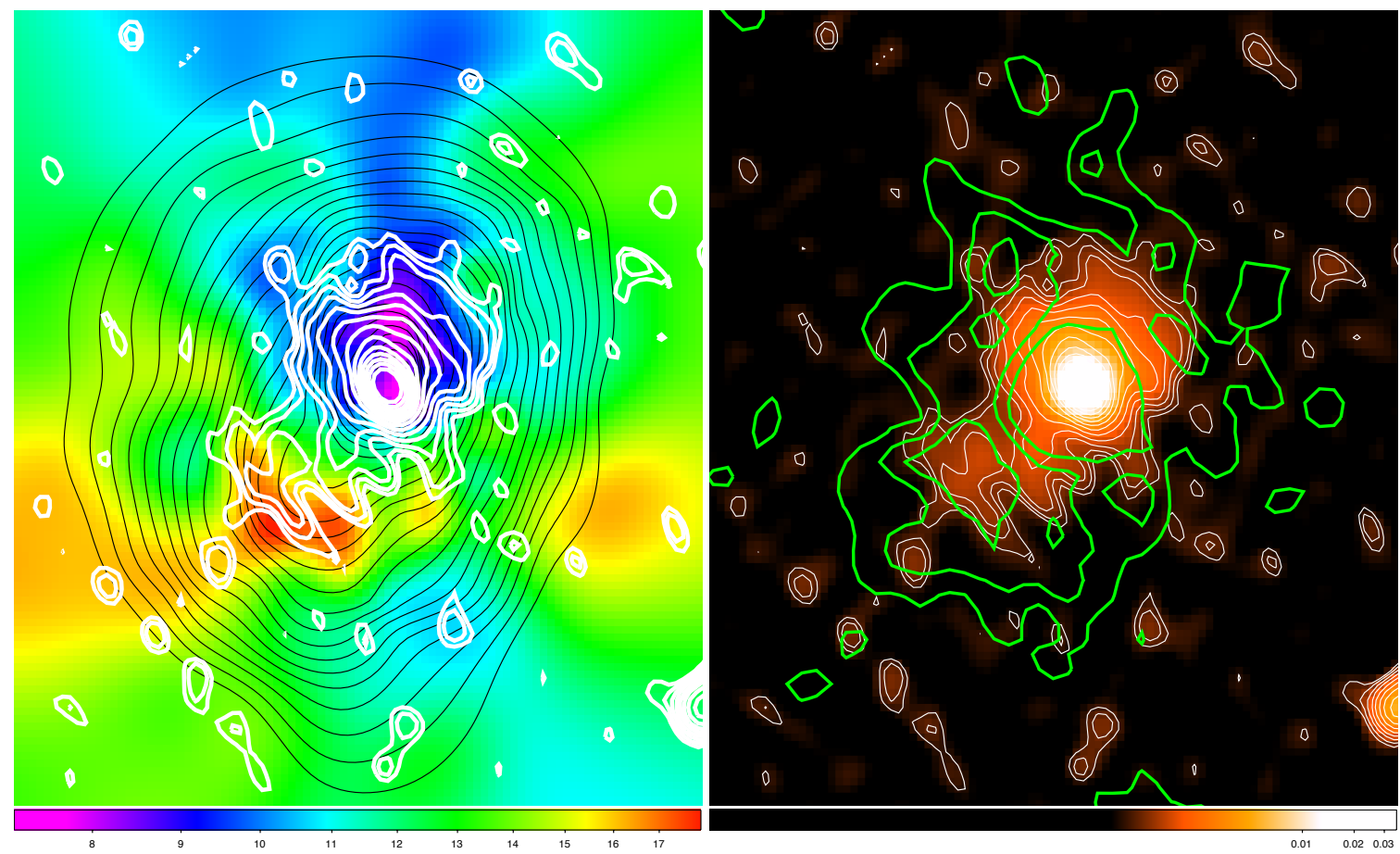

Figure 4: Left: XMM-Newton X-ray temperature map of the galaxy cluster RXJ1347 in keV. X-ray isocontours from the Chandra $[0.5,2.5] \mathrm{keV}$ band image are superimposed in black. Total intensity radio contours are overlaid in white. They start at $3 \sigma$ level and are spaced by a factor of $\sqrt{2}$. Right: total intensity $614 \mathrm{MHz}$ map and contours (white) of RXJ1347. Contours of the MUSTANG SZE image of the cluster are overlaid in green (levels as in Fig. 6 in Mason et al. (2010)). The shock region correspond to the inner contour on the SZ map and to the hottest (red) structure in the X-ray temperature map. Extracted from Ferrari et al. (2011).
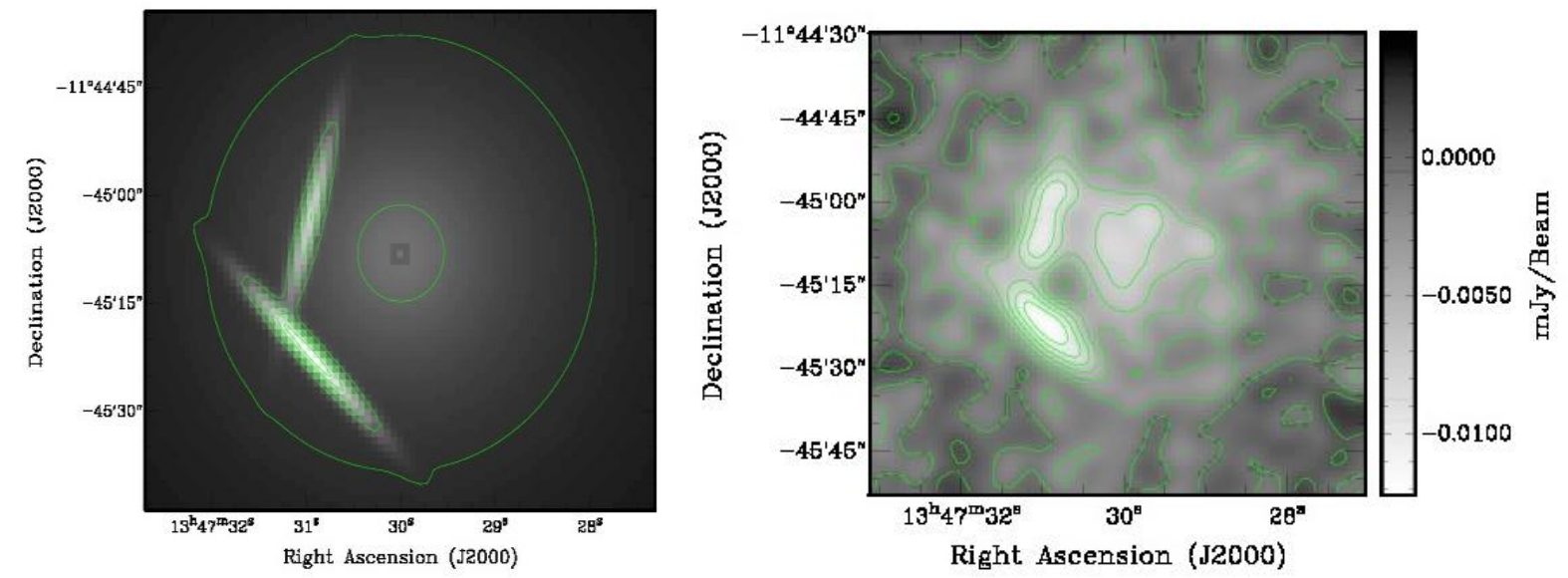

Figure 5: Left: Model of cluster RXJ1347 ( $\mathrm{z}=0.451)$ based on observations at $90 \mathrm{GHz}$ with the MUSTANG camera on GBT (Mason et al. 2010). Right: Simulated 1 hour SKA1-MID observation with a $20 \mathrm{k \lambda}$ uvtaper; the southern shock heated gas region is detected at $20 \sigma$. The detection of the bulk SZ effect from the cluster is at a significance of over $100 \sigma$ if mapped with a $5 k \lambda$ taper. 
baselines used for measure of the SZ. As a result, for the 1 hour simulation shown in Figure 3 the noise level from a map with a uv-taper chosen to give a psf of $1^{\prime \prime}$ is four times lower than that in the map which shows the SZ. Thus sources with flux $S \approx 3 \mu \mathrm{Jy}$ and above will be able to be identified and subsequently subtracted from the short baselines that measure the SZ. Extrapolating from the 10C source counts (Davies et al. 2011) we expect to detect around 1 source per square arcminute at this level in the field and approximately ten times as many in the centre of the cluster. Source confusion is therefore not an issue. The SKA1 aims to have dynamic range of $70 \mathrm{~dB}$ in order to address other science areas; since brightest cluster point sources are typically $S \approx 10-100 \mathrm{mJy}$, for SZ work we will not need anything like this performance.

In addition to the extended radio halos and relics continuum emission (Ferrari et al. 2015), galaxy clusters host a variety of extended radio sources, such as tailed radio galaxies whose shape is determined by interaction with the ICM (e.g. Giacintucci and Venturi (2009)); radio bubbles that create holes in the ICM distribution and rise buoyantly through the thermal gas (e.g. Dunn et al. (2005)); and compact radio sources related to galaxy activity (either blazar and/or starburst like). In order to study the diffuse SZ effect signal in clusters, it is of course crucial to be able to separate the different kinds of radio sources in the cluster environment, i.e. to discriminate between SZ and the radio emission related to active galaxies or to other non-thermal processes in the ICM.

Although these different radio sources have a wide variety of spectral shapes, they are all very distinct from the approximately thermal spectrum of the SZ effect (see Figure 1). Radio halo synchrotron spectra are usually quite steep (spectral slopes $\alpha_{r}>1$ ) and extended radio sources within the cluster (e.g., radiogalaxies with extended jets/lobes) also show quite steep spectra in their extended regions due to the effects of electron aging and radiative losses. Intra-cluster cavities have even steeper spectra ( $\alpha_{r} \approx 0.7-2.3$, see Birzan et al. (2004)) being filled with the termination region of radiogalaxy lobes that are usually populated by old electrons. A spatially-resolved spectral analysis with the sensitivity and resolution offered by the SKA1-MID will greatly help in providing the component separation needed to study the extended SZ effect signal in galaxy clusters. In addition, such spectral capability may also be able to distinguish any thermal and non-thermal component of the SZ effect in clusters through careful analysis of the Band 5 data from SKA1-MID.

Simulated SKA1-MID observations of non-thermal emission in galaxy clusters (Ferrari et al. 2015) demonstrate that the study of extended radio emission features in clusters is feasible, thanks to developments in deconvolution and source detection algorithms optimised for the analysis of extended and diffuse radio sources. With these developments, the SKA1-MID will allow multifrequency images of diffuse cluster radio sources to be made over large bands ranges (specifically Band 5), enabling detailed spectral index studies of galaxy clusters, which is essential for the component separation analysis and the detection of the SZ effect signal.

This analysis can be extended in the future to include i) a comparison between deconvolution results obtained using the new reconstruction algorithms based on compressed sensing and sparse representations (e.g. the MORESANE algorithm and the multi-scale version of CLEAN (Dabbech et al. 2014)), ii) polarisation studies for targeted observations, iii) an extended feasibility study taking into account the full SKA1 frequency range (including also SKA1-LOW), iv) a detailed analysis using the configuration of the full SKA array aimed at the study of the SZ effect in galaxy clusters. 


\section{SKA uniqueness and synergies for $\mathrm{SZ}$ observing}

As mentioned in Section 1 there are several telescopes that have been specifically designed for SZ work; the SKA1-MID will provide useful complementary capabilities to these. The SPT and ACT telescopes are primarily designed for surveying, enabled by their large fields of view, but they are unattractive for pointed observational programmes. Their angular resolution of $\approx$ $1^{\prime}$ is insufficient to image the detailed structure discussed in Section 3. SKA1-MID's resolution and sensitivity will be ideal for follow-up imaging of the cluster catalogues produced by these instruments. Similarly, Planck with its resolution of $\approx 5^{\prime}$ is a survey instrument for SZ. Its cluster catalogue has 1227 entries (Planck Collaboration XXIX 2013), but its cluster selection function is weighted more heavily towards low redshift than either SPT and ACT.

The SZA (Carlstrom 2006) (now part of the CARMA array) and AMI are centimetre wavelength interferometric telescopes that are ideal for SZ follow-up observations. While they cannot match SKA1-MID's sensitivity, their access to shorter baselines than those available to SKA1-MID are a useful complement, especially for studies of low redshift clusters.

CCAT (Woody et al. 2012) has resolution of a few arc-seconds and will be capable of the same type of observations proposed in this chapter for the SKA1-MID. Golwala (2008) calculates that a 600 hour programme on CCAT could study 100 clusters in the mass range $M=3.5 \times 10^{14}$ $1 \times 10^{15} M_{\odot}$; for comparison, in Section 2 we describe a 1000-hour SKA1-MID programme to follow up the 1000 clusters with $M_{200}>4 \times 10^{14} M_{\odot}$ in the high redshift eROSITA sample. CCAT's great strength is its unique frequency coverage, from $90 \mathrm{GHz}$ to above $1 \mathrm{THz}$, which give the potential for spectrally discriminating the thermal, kinematic and relativistic contributions to the SZ effect. Combining a low frequency 8.8-13.8 GHz measurement from SKA1-MID with the CCAT measurements can greatly help this discrimination (Knox et al. 2004).

ALMA (Brown et al. 2004) is potentially very powerful for SZ work. However, ALMA's field of view, even at Band 3, is small, less than $1^{\prime}$, and so it resolves out the bulk of the cluster SZ signal. For high resolution imaging of the detailed ICM structure ALMA is, however, very complementary to the SKA1-MID. As discussed in Scaife \& Grainge (2010), a possible future upgrade to ALMA through implementing Band 1 capabilities would greatly improve ALMA's utility as an SZ instrument.

\section{Early SKA1 science and looking towards the full SKA}

Pointed SZ observations are an attractive early SKA1-MID science goal; it has been shown that a great deal of good work could be performed with MeerKAT (Scaife et al. 2009). As has been discussed earlier, the SZ signal is detected primarily on the shorter SKA1-MID baselines, with some sensitivity required on longer baselines to remove radio contamination. So assuming a build-out from the centre, filling out the MeerKAT core to give good filling factor out to high radius greatly enhances the SKA1-MID's for SZ work. Also, the autocorrelations provided by the new SKA1-MID antennas give invaluable information about the zero-spacing flux.

Looking to SKA2, a possible increase in maximum observing frequency is extremely attractive for SZ measurements. Now that autocorrelations are an accepted part of the SKA Baseline, one can start to speculate whether the SKA2 will aim to fill the gap in uv-space between the auto- 
and cross-correlations; following the solution adopted by ALMA, this could be achieved with the addition of a close-packed array of small $(\sim 7 \mathrm{~m})$ dishes.

\section{Acknowledgments}

Chiara Ferrari acknowledges financial support by the "Agence Nationale de la Recherche" through grant ANR-09-JCJC-0001-01, the "Programme National Cosmologie et Galaxies (2014)", the $B Q R$ program of Lagrange Laboratory (2014). Sergio Colafrancesco acknowledges support by the South African Research Chairs Initiative of the Department of Science and Technology and National Research Foundation and by the Square Kilometre Array (SKA). Stefano Borgani acknowledges financial support from the PRIN-MIUR 2009AMXM79 Grant, from the PRIN-INAF 2012 Grant "The Italian network for computational cosmology" and from the INDARK INFN Grant. We would like to thank Dunja Fabjan for providing Figure 2. We thank Malak Olamaie for cluster models.

\section{References}

Allen, S. W., Evrard, A. E., \& Mantz, A. B. 2011, ARAA, 49, 409 [arXiv:1103.4829]

Arnaud, M., Pratt, G. W., Piffaretti, R., et al. 2010, A\&A, 517, A92 [arXiv: 0910.1234]

Basu, K., 2012, MNRAS, 421, 112, [arXiv:1111.2856]

Birkinshaw, M., Gull, S. F., and Hardebeck, H., 1984, Nature, 309, 34

Birkinshaw, M., 1999, Phys. Rept., 310, 97 [arXiv :astro-ph/9808050]

Birzan, L. et al. 2004, ApJ, 607, 800 [arXiv: astro-ph / 0402348 ]

Brown, R. L., Wild, W., Cunningham C., 2004, Advances in Space Research, 34, 555

Brunetti, G., \& Jones, T. W. 2014, International Journal of Modern Physics D, 23, 30007, [arXiv:1401.7519]

Carlstrom, J. E., Holder, G. P., and Reese, E. D., 2002, ARAA, 40, 643 [arXiv:astro-ph/0208192]

Carlstrom J. E., 2006, ASPC, 356, 35

Carlstrom, J. E. et al., Publications of the Astronomical Society of the Pacific, 2011, 123, 568 [arXiv:0907.4445]

Cassano, R. et el., 2013, ApJ, 777, 141, [arXiv:1306.4379]

Colafrancesco, S., Marchegiani, P. and Palladino, E. 2003, A\&A, 397, 27 [arXiv:astro-ph/0211649)]

Colafrancesco, S. et al. 2014, A\&A, 566, 42 [arXiv:1312 . 1846]

Dabbech, A., Ferrari, C., Mary, D., Slezak, E. and Smirnov, O., 2014, in preparation.

Davies, M. L. et al., 2011, MNRAS, 415, 2708, [arXiv: 1012.3659]

Dunn, R. J. H., Fabian, A. C., \& Taylor, G. B., 2005, MNRAS, 364, 1343 [arXiv:astro-ph/0510191]

Fabian, A.C., Sanders, J.S., Ettori, S., et al., 2000, MNRAS, 318, L65 [arXiv:astro-ph/0007456]

Ferrari, C., Govoni, F., Schindler, S., Bykov, A.M., Rephaeli, Y., 2008, SSRv, 134, 93, [arXiv:0801.0985] 
Ferrari, C., Intema, H.T., Orrú, E., et al., 2011, A\&A, 534, 12 [arXiv: 1107.5945 ]

Ferrari, C., et al., 2015, "Non-thermal emission from galaxy clusters: feasibility study with SKA", in "Advancing Astrophysics with the Square Kilometre Array", PoS(AASKA14)075

Feretti, L., Giovannini, G., Govoni, F., and Murgia, M., 2012, A\&ARv, 20, 54 [arXiv: 1205.1919]

Gitti, M., Ferrari, C., Domainko, W., Feretti, L., and Schindler, S., 2007, A\&A, 470, 25, [arXiv:0706.3000]

Giacintucci, S. and Venturi, T., 2009, A\&A, 505, 55 [arXiv:0907.2306]

Golwala, S., 2008, "CCAT Feasibility Study - SZ Science” http://www.astro.caltech.edu/ golwala/talks/CCATFeasibilityStudySZScience.pdf

Grainge, K., Jones, M., Pooley, G., Saunders, R., Edge, A., 1993, MNRAS, 265, 57

Jones, M. E. et al., 1993, Nature, 365, 320

Kay, S. T., Peel, M. W., Short, C. J., et al. 2012, MNRAS, 422, 1999 [arXiv: 1112 . 3769]

Knox, L., Holder, G. P., Church, S. E., 2004, ApJ, 612, 96 [arXiv: astro-ph / 0309643 ]

Le Brun, A. M. C., McCarthy, I. G., Schaye, J., \& Ponman, T. J. 2014, MNRAS, 441, 1270 [arXiv:1312.5462]

Kravtsov, A. V., \& Borgani, S. 2012, ARAA, 50, 353 [arXiv:1205.5556]

M. Markevitch, Intergalactic shock fronts, SSRv 134 (2008) 93, [arXiv: 1010 . 3660]

Marrone, D. P., Smith, G. P., Okabe, N., et al. 2012, ApJ, 754, 119 [arXiv: 1107.5115 ]

Mason, B.S., Dicker, S.R., Korngut, P.M., et al., 2010, ApJ, 716, 739

Merloni, A. et al., 2012, "eROSITA Science Book"

Nagai, D., Kravtsov, A. V., Vikhlinin, A., 2007, ApJ, 668, 1 [arXiv:astro-ph/0703661]

Perrott, Y. C. et al., 2014, [arXiv1405.5013]

Pillepich, A., Porciani, C., \& Reiprich, T. H. 2012, MNRAS, 422, 44, [arXiv:1111. 6587]

Planck Collaboration: Ade, P. A. R. et al, Astronomy \& Astrophysics, 2011, 536, 8 [arXiv:1101.2024]

Planck Collaboration, Ade, P. A. R., Aghanim, N., et al. 2013, [arXiv:1303.5080]

Planck Collaboration: Ade, P. A. R. et al., 2013, "Planck 2013 results. XXIX. Planck catalogue of Sunyaev-Zeldovich sources”, [arXiv: 1303.5089]

Planelles, S., Borgani, S., Fabjan, D., et al. 2014, MNRAS, 438, 195 [arXiv: 1311.0818 ]

Rozo, E. et al., 2009, ApJ, 699, 768 [arXiv: 0809.2794 ]

Scaife, A. M. M. et al., 2009, COSMOKatSZ science proposal.

Scaife, A. M. M., \& Grainge, K. J. B., 2010, BASI, 38, 185 [arXiv: 1002 . 1895]

Sembolini, F., Yepes, G., De Petris, M., et al. 2013, MNRAS, 434, 2718

Sun, M., Sehgal, N., Voit, G. M., et al. 2011, ApJ, 727, L49 [arXiv: 1012 . 0312]

Sunyaev, R. A. and Zel'dovich, Y. B., 1970, Astroph. Sp. Sci., 7, 3

Sunyaev, R. A. and Zel'dovich, Y. B., 1972, Comments on Astrophysics and Space Physics, 4, 173

Swetz, D. S. et al., 2011, ApJS, 194, 41 [arXiv: 1007.0290 ]

Woody, D. et al., 2012, in Ground-based and Airborne Telescopes IV, edited by L. M. Stepp, R. Gilmozzi, and H. J. Hall, vol. 8444 of Proc. SPIE, 84442M

Zwart, J. T. L. et al., 2008, MNRAS, 391, 1545 [arXiv:0 807.2469 ] 